\title{
QUEEN'S
UNIVERSITY
BELFAST
}

\section{Angiotensin receptor blocker use and gastro-oesophageal cancer survival: a population-based cohort study}

Busby, J., McMenamin, U., Spence, A., Johnston, B. T., Hughes, C., \& Cardwell, C. (2018). Angiotensin receptor blocker use and gastro-oesophageal cancer survival: a population-based cohort study. Alimentary Pharmacology and Therapeutics, 47(2), 279-288. https://doi.org/10.1111/apt.14388

Published in:

Alimentary Pharmacology and Therapeutics

Document Version:

Peer reviewed version

Queen's University Belfast - Research Portal:

Link to publication record in Queen's University Belfast Research Portal

Publisher rights

Copyright 2017 Wiley. This work is made available online in accordance with the publisher's policies. Please refer to any applicable terms of use of the publisher.

\section{General rights}

Copyright for the publications made accessible via the Queen's University Belfast Research Portal is retained by the author(s) and / or other copyright owners and it is a condition of accessing these publications that users recognise and abide by the legal requirements associated with these rights.

Take down policy

The Research Portal is Queen's institutional repository that provides access to Queen's research output. Every effort has been made to ensure that content in the Research Portal does not infringe any person's rights, or applicable UK laws. If you discover content in the Research Portal that you believe breaches copyright or violates any law, please contact openaccess@qub.ac.uk. 
Title: Angiotensin receptor blocker use and gastro-oesophageal cancer survival: a population-based cohort study

Authors: John Busby ${ }^{1}$, Úna McMenamin ${ }^{1}$, Andrew Spence ${ }^{1}$, Brian T Johnston ${ }^{2}$, Carmel Hughes ${ }^{3}$, Chris R Cardwell ${ }^{1}$

${ }^{1}$ Centre for Public Health, Queen's University Belfast, Belfast, UK

${ }^{2}$ Belfast Health and Social Care Trust, Belfast, Northern Ireland, United Kingdom

${ }^{3}$ School of Pharmacy, Clinical and Practice Research Group, Queen's University Belfast

Running title: ARBs and gastro-oesophageal cancer survival

Correspondence: Dr. John Busby, Centre for Public Health, Queen's University Belfast, Institute for Clinical Science, Royal Victoria Hospital, Grosvenor Road, Belfast, BT12 6BJ, +442890971640, john.busby@qub.ac.uk

Manuscript word count (including references): 4,640

Summary word count: 244

Number of figures: 1

Number of tables: 3

Number of supplementary files: 4 


\section{Summary}

Background: Angiotensin receptor blockers (ARBs; including candesartan, losartan, olmesartan and valsartan) are widely used to treat hypertension, heart failure and diabetic neuropathy. There is considerable preclinical evidence that ARBs can reduce cancer progression, particularly for gastric cancer. Despite this, epidemiological studies have yet to assess the impact of ARB use on gastrooesophageal cancer survival.

Aim: To investigate the association between post-diagnosis ARB use and gastro-oesophageal cancer survival.

Methods: We selected a cohort of patients with newly-diagnosed gastro-oesophageal cancer between 1998 and 2012 from English cancer registries. We linked to prescription and clinical records from the Clinical Practice Research Datalink, and to death records from the Office for National Statistics. We used time-dependant Cox-regression models to calculate hazard ratios (HRs) comparing gastro-oesophageal cancer-specific mortality between post-diagnosis ARB users and nonusers, after adjusting for demographics, comorbidities and post-diagnosis aspirin or statin use.

Results: Our cohort included 5,124 gastro-oesophageal cancer patients, of which 360 used ARBs, and 3,345 died due to their gastro-oesophageal cancer during follow-up. After adjustment, ARB users had moderately lower risk of gastro-oesophageal cancer mortality than the non-users (HR=0.83, 95\% $\mathrm{Cl}$ : $0.71,0.98)$. There was evidence of a dose-response relationship with the lowest HRs observed among patients receiving at least two years of prescriptions (HR=0.42, $95 \% \mathrm{Cl}: 0.25,0.72$ ).

Conclusions: In this large population-based gastro-oesophageal cancer cohort, we found moderately reduced cancer-specific mortality among ARB users. However, confirmation in further independent epidemiological studies with sufficient staging information is required. 
1

2 Oesophageal and gastric cancer are among the most common cancers in the world, with around 3

\section{Introduction} 456,000 and 952,000 new cases diagnosed annually. ${ }^{1}$ Prognosis is extremely poor, even in developed countries such as the United Kingdom, where $55 \%$ of patients die within one year of diagnosis. ${ }^{2}$ Those who survive suffer a marked reduction in their quality of life during treatment and recovery. ${ }^{3,4}$ (1)

Angiotensin receptor blockers (ARBs; including candesartan, losartan, olmesartan and valsartan) are widely used and effective treatments for hypertension, heart failure and diabetic neuropathy. ${ }^{5,6}$ In England, ARBs are recommended as a first-line pharmacological treatment for hypertension patients aged under fifty-five or with comorbid diabetes, ${ }^{7,8}$ and nearly 20 million prescriptions are dispensed annually. ${ }^{9}$ An estimated 200 million patients are treated with ARBs worldwide, representing $25 \%$ of all antihypertensive agents. ${ }^{10}$ ARBs reduce blood pressure by blocking angiotensin II type I receptors within the renin-angiotensin system, however evidence of local expression of renin-angiotensin system components within cancer cells ${ }^{11,12}$ has fuelled debate that they might also affect cancer tumour development. ${ }^{11,}{ }^{13}$ In-vitro and mouse models at various cancer sites have shown that ARBs reduce tumour growth, stimulate cell apoptosis, reduce metastasis, and inhibit angiogenesis, suggesting that several chemopreventive mechanisms are possible. ${ }^{14-18}$ More specifically, ARBs have been shown to slow proliferation, inhibit fibrosis, and prevent stress-induced injury in gastric cancer cell line and animal model studies. ${ }^{19-23}$

Despite convincing preclinical evidence that ARBs could influence cancer risk and progression, studies in humans are inconsistent. A meta-analysis of secondary outcomes from randomised controlled trials found little evidence of an association between ARB use and cancer risk ${ }^{24}$, while a meta-analysis of observational studies reported reduced cancer risk among long-term ARB users. ${ }^{25}$ Observational studies of cancer progression are fewer, but have found improved outcomes among ARB or angiotensin converting enzyme inhibitor (an alternative antihypertensive medication which 
27 also inhibits the renin-angiotensin system) users across several cancer sites, while also highlighting

28 methodological issues with the current literature such as poor generalisability, short follow-up,

29 inadequate case-mix adjustment and potential exposure misclassification. ${ }^{26,27}$ To date, no studies

30 have investigated ARB use and gastro-oesophageal mortality, although olmesartan has been shown

31 to cause severe enteropathy in some patients, suggesting upper-gastrointestinal effects. ${ }^{28}$

32 Consequently, we used a large population-based dataset from the UK to robustly assess this

33 association. 
Our study used data from the English National Cancer Data Repository, linked to GP records from the

37 UK Clinical Practice Research Datalink, deprivation indices from census information, and death registration data from the Office for National Statistics . The National Cancer Data Repository holds UK-wide data from English cancer registries compiled from general practices, National Health Service and private hospitals, and death certificates. It contains detailed information about the patient's cancer, including year of diagnosis, stage, histologic grade, tumour type (adenocarcinoma or squamous cell carcinoma) and treatment (surgery, chemotherapy, and radiotherapy). The Clinical Practice Research Datalink contains computerised medical records from 674 general practices (approximately $7 \%$ of the UK population) which are audited for data completeness and quality. Practices meeting a predefined quality threshold are deemed 'up to standard' and included in future extracts. Data recorded within the Clinical Practice Research Datalink include patient demographics, clinical diagnoses (using Read codes) and prescription medication use. Previous research has found prescription and clinical information to be of high quality. ${ }^{29}$ Office for National Statistics deathregistration data provide details on the date and cause(s) of death.

Ethical approval for all purely observational research using anonymised Clinical Practice Research Datalink data was obtained from a National Research Ethics Service Committee. The protocol for this study was approved by the Clinical Practice Research Datalink Independent Scientific Advisory Committee (Ref: 15_096R), and has been made available to reviewers.

57 We identified a cohort of patients with newly-diagnosed gastro-oesophageal cancer from English cancer registry records (ICD-10 codes C15 or C16) between 1998 and 2012. Cohort members with a 
previous diagnosis of cancer (excluding non-melanoma skin cancer) were identified and excluded using a list of cancer Read codes modified for use in the Clinical Practice Research Datalink. ${ }^{30}$ Patients were excluded if they were diagnosed: (a) before they were registered with a Clinical Practice Research Datalink practice, (b) before their practice was deemed up to research standard, (c) after they left a Clinical Practice Research Datalink practice, or (d) after data were last collected from their practice by the Clinical Practice Research Datalink. A small number of patients had more than one gastro-oesophageal cancer record in the National Cancer Data Repository; when this occurred we used their first record.

67

Deaths were identified from Office for National Statistics records, and gastro-oesophageal cancer specific deaths were defined as those with an underlying cause of gastro-oesophageal cancer (ICD10 codes C15, C16 or C26). Patients with less than six months follow-up were excluded as it is unlikely that these could be influenced by post-diagnosis medication use. Therefore the follow-up period started from six months after diagnosis. The end of follow-up was the earliest date of: death, end of registration with the practice, last collection of data from the practice, or end of death record follow-up.

\section{Definition of exposure}

We used the British National Formulary ${ }^{31}$ to compile a list of proprietary and generic medication names to identify ARB use (Appendix 1). We added a lag of six months to ARB use as these medications are unlikely to have an immediate effect on gastro-oesophageal cancer progression, and to prevent reverse causation. ${ }^{32,33}$ A diagram illustrating our design is shown in Appendix 2. We defined patients as users after they received their first prescription during the exposure period. To enable the testing of dose-response relationships we extracted data on the number of tablets and medication strength, and calculated defined daily doses (DDDs). The DDD system is a validated measure of drug consumption maintained by the World Health Organisation. A single DDD is the 
average maintenance dose per day of a drug used for its main indication in adults (e.g. hypertension for ARBs). There was insufficient information to calculate DDDs for $0.1 \%$ of prescriptions, and implausible values were recorded in a further $0.1 \%$. In these cases we assumed the most common DDD based on other prescriptions with complete information. We calculated a running DDD total for each patient and identified the day when patients received their $1^{\text {st }}$ (first use), 183th (six months' use), $365^{\text {th }}$ (one year's use) and $730^{\text {th }}$ (two years' use) DDDs.

91

92

\section{Covariates}

Patients' age, smoking (current, former, never), units of alcohol consumed per week (0,1-14, 15-28, $29-42,43+$ ), and body mass index (BMI; underweight [BMl<18.5], normal weight $[18.5 \leq \mathrm{BMI}<25]$, overweight $[25 \leq \mathrm{BMI}<30]$ and obese $[\mathrm{BMI} \geq 30])$ data were determined from the closest GP record before gastro-oesophageal cancer diagnosis (values more than 10 years before diagnoses were discarded). We used GP records to identify pre-diagnosis comorbidities (cerebrovascular disease, chronic pulmonary disease, congestive heart disease, diabetes, liver disease, myocardial infarction, peptic ulcer disease, peripheral vascular disease, renal disease) using a list of Read codes modified for use in the Clinical Practice Research Datalink. ${ }^{30}$ Deprivation data were available from census information, and based on the 2010 Index of Multiple Deprivation score of the patient's postcode. We used Clinical Practice Research Datalink prescription records to identify patients using statins or aspirin after diagnosis, as these have been shown to influence cancer progression previously. ${ }^{34,35}$

We calculated descriptive statistics and compared the demographic, lifestyle and clinical characteristics of the ARB users and non-users. We produced survival graphs using the SimonMakuch method, which is an alternative to the Kaplan-Meier but appropriately accounts for timevarying covariates. ${ }^{36}$ We used time-dependant Cox regression models to calculate hazard ratios (HRs) comparing gastro-oesophageal cancer-specific death between ARB users and non-users. We 
conducted analyses for gastro-oesophageal cancer, and separately for oesophageal (ICD-10 code

112 C15) and gastric (ICD-10 code C16) cancer. In our primary analysis we included ARB use as a timevarying covariate to avoid immortal time bias. ${ }^{37}$ Therefore patients were initially included within the analysis as non-users until six months after their first use (due to the exposure lag), after which they were included as users until the end of follow-up. Our primary analysis adjusted for age at diagnosis, year of diagnosis (separate term for each year), deprivation quintile, comorbidities (separate terms for each), post-diagnosis use of aspirin or statins (using time-varying covariates and a six month lag after their first prescription), cancer site (gastric or oesophageal), and treatment within six months of diagnosis (separate terms for surgery, chemotherapy, radiotherapy). We repeated our analysis by number of DDDs prescribed (e.g. patients were included in the 1-364 DDD group until six months after they received their $365^{\text {th }}$ DDD), and for candesartan and losartan, the most commonly prescribed ARBs. ${ }^{9}$ Again, the use of time-varying covariates negates immortal time bias. We conducted interaction tests to assess differences by tumour type.

\section{Sensitivity and subgroup analyses}

We conducted sensitivity analysis for all-cause mortality, and for cause-specific mortality where deaths with a secondary cause (i.e. listed as an 'other cause of death' on death certificate) of gastrooesophageal cancer were included. We also conducted sensitivity analyses with a lag period of zero (patients followed-up from diagnosis) and twelve months (patients followed-up from twelve months after diagnosis). We performed two simplified analyses which controlled for immortal time bias without time-varying covariates. ${ }^{37}$ Firstly, we based ARB usage on the six months after diagnosis, and followed-up patients from six months after diagnosis. Secondly, we investigated ARB usage in the year prior to diagnosis, and followed-up patients from the date of diagnosis. Diagrams illustrating the design of our sensitivity analyses which vary the exposure lag and/or period are given in Appendix 2. We conducted subgroup analysis by tumour type (i.e. adenocarcinoma and squamous cell carcinoma), as these differ in incidence, risk factors and pathogenesis. We also carried out sub- 
group analysis restricted to patients receiving surgery, as they are likely to form a more homogenous group of earlier-stage patients.

To assess if confounding by indication was driving our results, we conducted three further sensitivity analyses restricted to patients with similar clinical diagnoses. First, we restricted our analysis to patients with a hypertension diagnosis (Read code categories G20 and 662) in the year prior to cancer diagnosis. Second, we restricted our analysis to patients who received an antihypertensive medication (diuretics, vasodilator antihypertensive drugs, centrally acting antihypertensive drugs, alpha-adrenoceptor blocking drugs, beta-blockers, angiotensin converting enzyme [ACE] inhibitors, ARBs, renin inhibitors, and calcium channel blockers) in the year prior to cancer diagnosis. Third, we compared patients who received ARBs to those who received a different antihypertensive medication after diagnosis (using a time-varying covariate), as the use of an active comparison can overcome several common pharmacoepidemiological biases. ${ }^{38}$ Similarly, we conducted negative control analyses ${ }^{39}$ for ACE inhibitors as they have similar indications to ARBs, but a distinct biological mechanism within the renin-angiotensin system. Therefore, if confounding was driving our ARB analyses we would expect to see similar associations for ACE inhibitors. Conversely, findings of a substantial association for ARBs, which are not replicated among the negative controls, would support a causal interpretation.

We performed additional sensitivity analysis adjusting for tumour prognostic features (stage, grade) and patient lifestyle factors (smoking, alcohol consumption, BMI) using multiple imputation with chained equations. Briefly, this is a simulation-based approach for handling missing data which leads to valid statistical inferences under certain assumptions. ${ }^{40}$ The imputation used ordered logit models with age, deprivation, death indicator and the baseline hazard function as covariates. Lastly, we used the Fine and Gray sub-distribution hazard model to assess the impact of competing risks from non-gastro-oesophageal cancer deaths. ${ }^{41}$ 
We identified 9,714 gastro-oesophageal cancer cases with no prior cancer diagnosis registered at

Clinical Practice Research Datalink practices. We excluded 4,590 patients as they had either less than six months follow-up $(n=4,582)$ or a duplicate record in the National Cancer Data Repository $(n=8)$, leaving 5,124 patients for analysis. Median follow-up was 1.4 years (maximum 17.2 years). ARB users were more likely to be female, have comorbidities (particularly diabetes, renal disease and congestive heart disease), be treated with statins or aspirin after diagnosis, undergo surgery, be nonor ex-smokers, and be obese (Table 1).

\section{Association between ARB use and survival}

Overall, ARB users were at a moderately lower risk of gastro-oesophageal cancer death than nonusers both before (unadjusted $\mathrm{HR}=0.80,95 \% \mathrm{Cl}$ : 0.69, 0.94; Figure 1) and after adjustment for demographics, comorbidities, cancer treatments and post-diagnosis aspirin or statin use (adjusted $\mathrm{HR}=0.83,95 \% \mathrm{Cl}: 0.71,0.98$; Table 2). There was evidence of a dose-response relationship ( $\mathrm{p}$-value for trend=0.003); the largest differences in mortality were found among those who had received at least $730 \mathrm{DDDs}$ (adjusted $\mathrm{HR}=0.42,95 \% \mathrm{Cl}: 0.25,0.72$ ), although associations were relatively similar for those receiving 1-182, 183-364, 365-729 DDDs. We observed broadly similar estimates for losartan (adjusted $\mathrm{HR}=0.84,95 \% \mathrm{Cl}: 0.59,1.21$ ) and candesartan (adjusted $\mathrm{HR}=0.73,95 \% \mathrm{Cl}: 0.49$, 1.09). We found slightly stronger association among patients with gastric (adjusted $\mathrm{HR}=0.79,95 \% \mathrm{Cl}$ : $0.62,1.00$ ) than oesophageal (adjusted $\mathrm{HR}=0.89,95 \% \mathrm{Cl}: 0.71,1.10$ ) cancer, although the doseresponse patterns were comparable to the combined analysis, with very large decreases in cancerspecific mortality among both oesophageal (adjusted HR=0.44, 95\% Cl: $0.21,0.94$ ) and gastric (adjusted HR=0.40, 95\% Cl: $0.19,0.84$ ) patients receiving at least 730 DDDs of ARBs. The full results of the gastro-oesophageal model are given in Appendix 3. 
190 Our results were similar in the simpler analysis basing ARB use on the year prior, or six-months after

191 diagnosis (Table 3). Our conclusions were unchanged when expanding our cancer-specific death definition to include secondary death causes, and for all-cause mortality. Our results were robust to alterations in the exposure lag period from zero to twelve months, and did not change appreciably when adjusting for tumour prognostic features (i.e. stage, grade), or patient lifestyle factors (i.e. smoking, alcohol, BMI) using multiple imputation methods.

196

We observed broadly similar hazard ratios when restricting our analysis to patients who received surgical treatment (adjusted $\mathrm{HR}=0.81 ; 95 \% \mathrm{Cl}: 0.63,1.05$ ), or with a prior diagnosis of hypertension (adjusted HR=0.83; 95\% Cl: 0.62, 1.09). Likewise, our results were broadly similar when restricting to those in receipt of antihypertensive medications before diagnosis (adjusted $\mathrm{HR}=0.80 ; 95 \% \mathrm{Cl}: 0.67$, 0.94), or when comparing ARB users to patients receiving a different antihypertensive medication after gastro-oesophageal cancer diagnosis (adjusted $\mathrm{HR}=0.83 ; 95 \% \mathrm{Cl}: 0.71,0.98$ ). We did not find any evidence of an association between ACE inhibitor use and gastro-oesophageal survival (adjusted HR: $0.98,95 \% \mathrm{Cl}: 0.89,1.08$; Appendix 4). The association between ARB use and cancer-mortality was slightly stronger for patients with adenocarcinoma (adjusted $\mathrm{HR}=0.78,95 \% \mathrm{Cl}: 0.65,0.94$ ) than squamous cell carcinoma (adjusted $\mathrm{HR}=0.95,95 \% \mathrm{Cl}: 0.63,1.43$ ), although this difference was not statistically significant ( $p$-value for interaction $=0.52$ ). 
210 In this large, population-based cohort of newly-diagnosed gastro-oesophageal cancer patients, we

211 found a statistically significant reduction of $17 \%$ in cancer-specific morality among ARB users after

212 adjustment for patient demographics, comorbidities, cancer treatments and post-diagnosis aspirin or statin use. There was some evidence of a dose-response relationship with the largest decreases in mortality observed among patients receiving at least 2 years' worth of prescriptions.

\section{Strengths and weaknesses}

This is the first study to investigate ARB use and survival from gastro-oesophageal cancer. Our study

is based on a high-quality population-based cohort of patients with registry-confirmed gastrooesophageal cancer which was followed-up for up to 17 years. ${ }^{29}$ Linkage to Office for National

Statistics death registration data allowed robust verification of death, and facilitated a gastrooesophageal cancer-specific analysis, which should be more sensitive to small changes in diseasespecific mortality, and less susceptible to confounding by indication than all-cause deaths. ${ }^{33,42}$ Although some misclassification of death cause is possible, studies have shown this is likely to have a limited impact on our estimates (as there is no obvious mechanism for differential misclassification $)^{43}$, and our results were similar when including deaths where gastro-oesophageal cancer was not the underlying cause. We used prescribing data collected as part of routine clinical care which accurately reflects GP prescribing practices and negates the risk of recall bias. These data also included detailed information on the type of $A R B$, and the strength, quantity and timing of prescription, which allowed us to investigate dose-response relationships, and conduct separate analyses for specific medications. ARBs are not available over-the-counter in the UK, which negates exposure misclassification due to over-the-counter usage. 
Our study had several potential weaknesses. We necessarily excluded patients who lived for less than six months after diagnosis, therefore our results cannot be applied to those with a very poor prognosis. Our study is observational and hence open to confounding by incomplete or unmeasured covariates. Although we have adjusted for several key determinants of gastro-oesophageal cancer survival (e.g. age, comorbidities and cancer treatments), some were incompletely recorded (e.g. smoking history) and others were not available within our dataset (e.g. ethnicity and family history). The lack of complete information on cancer stage is of particular concern, especially as ARB users were more frequently diagnosed with lower-stage cancers than non-users (e.g. $16.9 \%$ vs. $9.4 \%$ stage 1). It is also possible that our study could be subject to a 'healthy user effect' whereby patients who receive one preventative therapy (ARBs) are more likely to use other therapies (e.g. endoscopy), or more closely follow medical advice (e.g. attend medical appointments). ${ }^{44}$

Nevertheless, the findings from our sensitivity analyses suggest that confounding or missing data issues were not solely driving our results. For example, the protective association for ARBs was preserved when using other antihypertensive medications as an active comparator, when restricting to those who received surgery (who should form a more homogeneous cohort of lower-stage patients), and when limiting our analysis to patients with a prior hypertension diagnosis. Similarly, we observed little evidence of an association between gastro-oesophageal cancer mortality and ACE inhibitor use, which have similar indications to ARBs. Lastly, our conclusions were unchanged when using multiple imputation to adjust for cancer stage and grade, albeit this analysis is particularly sensitive to departures from the 'missing at random' assumption due to the large proportion of missing data for these variables. ${ }^{45}$ We do not know if patients adhered to their prescribed medications, however our main conclusions were similar when restricting our analysis to patients who received multiple ARB prescriptions ( $\geq 730$ DDDs), where non-compliance is less of a concern. Finally, ARBs were one of six medications investigated within a broader programme of work, meaning that multiple testing could be a potential concern. 
We are unaware of any other studies comparing gastro-oesophageal cancer mortality between ARB users and non-users. Several studies have previously investigated the role of renin-angiotensin system blockade on cancer survival. However they have generally combined ACE inhibitors with $A R B s^{27}$, potentially obfuscating the therapeutic effect of each medication as they have distinct mechanisms of action within the renin-angiotensin system, and are known to differ in their side effect profiles. Our finding of a much stronger association with gastro-oesophageal survival among ARB than ACE inhibitor users could suggest that separate analyses are indeed preferable.

Nevertheless, our results are consistent with findings of longer survival among gastro-oesophageal cancer patients using renin-angiotensin system blockade medications in two other studies based in Taiwan and Korea. ${ }^{46,47}$ However, each of these were substantially limited by poor generalisability (e.g. restricted to advanced gastric cancer), an inability to identify cancer-specific deaths, inappropriate statistical methods (e.g. chi-squared test) and small sample size (196 patients in total). Our study improves on the current literature by using appropriate methodology to analyse a population-based cohort over thirty times larger than previous work. Our results are also consistent with the findings from several other observational studies reporting improved survival among reninangiotensin system blockade medication users at other sites. ${ }^{26,}{ }^{27}$ For example, one recent metaanalysis found that mortality was $25 \%(95 \% \mathrm{Cl}: 1,43)$ lower among cancer patients using ACE inhibitors or ARBs, with particularly large decreases in urinary tract, colorectal, pancreatic, and prostate cancer. ${ }^{27}$

\section{Implications for practitioners and researchers}

Our results provide epidemiological evidence that the use of ARBs may be associated with improved gastro-oesophageal cancer survival. Our conclusions are consistent with preclinical research which 
has demonstrated that ARBs can slow tumour growth, stimulate cell apoptosis, reduce metastasis, and inhibit angiogenesis. ${ }^{14-18}$ More specifically, ARBs have been shown to slow proliferation, inhibit fibrosis, and prevent stress-induced injury in gastric cancer cell line and animal model studies. ${ }^{19-23}$ Our finding of a slightly stronger association among gastric than oesophageal cancer patients requires further exploration, however it could be due to higher renin-angiotensin system expression among patients with H.pylori infection ${ }^{48}$, the most important risk factor for gastric cancer. Likewise our finding of a slightly stronger association for adenocarcinoma than squamous cell carcinoma could be due to ARBs promoting healing of reflux oesophagitis among proton pump inhibitor users. ${ }^{49}$

Our study suggests that it is worth further exploring the potential for ARBs to be repurposed as a gastro-oesophageal cancer treatment, particularly as they are inexpensive (losartan costs $f 1.15$ [\$1.48] per 28-tablet pack $)^{50}$, have no major safety concerns ${ }^{51}$, and are well tolerated by patients. ${ }^{52}$ In this paper we have demonstrated that the association with gastro-oesophageal cancer mortality adheres to several of Hill's criteria for causation including biological plausibility, experimental evidence, temporality, biological gradient, consistency and specificity. ${ }^{53}$ However, these findings should be replicated in independent epidemiological studies with more complete information on cancer stage.

\section{Conclusions}

In this large population-based cohort of patients with registry-confirmed gastro-oesophageal cancer, we found a $17 \%$ reduction in cancer-specific mortality among ARB users. Although this association adheres to several of Hill's criteria for causation, and is consistent with preclinical evidence, further independent epidemiological studies with more complete stage data is required. 
309 Acknowledgements: This study is based in part on data from the Clinical Practice Research Datalink 310 obtained under licence from the UK Medicines and Healthcare products Regulatory Agency. 311 However, the interpretation and conclusions contained in this study are those of the author/s alone.

312 Access to the Clinical Practice Research Datalink dataset was funded by a Clinical Research Bursary 313 for AS from Cancer Research-UK [C54914/A20558].

314

315 Abbreviations: ACE: angiotensin converting enzyme; ARB: angiotensin receptor blocker; BMI: body 316 mass index; DDD: defined daily dose; HR: hazard ratio

Authorship Statement: CC, AS, BJ and CH conceived the study. JB conducted the analysis and drafted 319 the initial manuscript. All authors critically revised the article for intellectual content and approved 320 the final manuscript. JB acts as the study guarantor.

Conflict of interest: None to declare 
324 Figure Legends

325 Figure 1: Red line represents the proportion of ARB users that are alive at a given time after 326 diagnosis. Blue line represents the proportion of non-ARB users that are alive at a given time after 327 diagnosis. 


\section{References}

1. GLOBOCAN. Estimated Cancer Incidence, Mortaility and Prevelence Worldwide in 2012.

2. Office for National Statistics. Cancer survival in England: Patients diagnosed between 2010 and 2014 and followed up to 2015. 2016; Available from: http://www.ons.gov.uk/peoplepopulationandcommunity/healthandsocialcare/conditionsanddisease s/bulletins/cancersurvivalinenglandadultsdiagnosed/2010and2014andfollowedupto2015\#5-yearsurvival.

3. Viklund $P$, Wengstrom $Y$, Rouvelas $I$, et al. Quality of life and persisting symptoms after oesophageal cancer surgery. Eur J Cancer. 2006;42(10):1407-14.

4. Zieren $\mathrm{HU}$, Jacobi CA, Zieren J, et al. Quality of life following resection of oesophageal carcinoma. Br J Surg. 1996;83(12):1772-5.

5. Lindholm LH, Ibsen H, Dahlof B, et al. Cardiovascular morbidity and mortality in patients with diabetes in the Losartan Intervention For Endpoint reduction in hypertension study (LIFE): a randomised trial against atenolol. Lancet. 2002;359(9311):1004-10.

6. Pfeffer MA, Swedberg K, Granger CB, et al. Effects of candesartan on mortality and morbidity in patients with chronic heart failure: the CHARM-Overall programme. Lancet (London, England). 2003;362(9386):759-66. Epub 2003/09/19.

7. National Institute for Health and Care Excellence (NICE). Hyptertension in adults: diagnosis and management. 2016 [cited 2017 10/04/17]; Available from: https://www.nice.org.uk/guidance/cg127/chapter/2-Research-recommendations.

8. National Institute for Health and Care Excellence (NICE). Type 1 diabetes in adults: diagnosis and management. 2016 [cited 2017 10/04/17]; Available from: https://www.nice.org.uk/guidance/ng17/chapter/1-Recommendations.

9. Powell-Smith A, Goldacre B. OpenPrescribing.net. 2016. Available from: https://openprescribing.net/.

10. Volpe $M$, Azizi $M$, Danser $A H J$, et al. Twisting arms to angiotensin receptor blockers/antagonists: the turn of cancer. European Heart Journal. 2011;32(1):19-22.

11. Deshayes F, Nahmias C. Angiotensin receptors: a new role in cancer? Trends in endocrinology and metabolism: TEM. 2005;16(7):293-9. Epub 2005/08/03.

12. Garg M, Angus PW, Burrell LM, et al. Review article: the pathophysiological roles of the renin-angiotensin system in the gastrointestinal tract. Alimentary pharmacology \& therapeutics. 2012;35(4):414-28. Epub 2012/01/10.

13. George AJ, Thomas WG, Hannan RD. The renin-angiotensin system and cancer: old dog, new tricks. Nat Rev Cancer. 2010;10(11):745-59.

14. Rocken C, Lendeckel U, Dierkes J, et al. The number of lymph node metastases in gastric cancer correlates with the angiotensin I-converting enzyme gene insertion/deletion polymorphism. Clinical cancer research : an official journal of the American Association for Cancer Research. 2005;11(7):2526-30. Epub 2005/04/09.

15. Neo JH, Malcontenti-Wilson C, Muralidharan V, et al. Effect of ACE inhibitors and angiotensin II receptor antagonists in a mouse model of colorectal cancer liver metastases. J Gastroenterol Hepatol. 2007;22(4):577-84. 
16. Gong $\mathrm{Q}$, Davis $\mathrm{M}$, Chipitsyna $\mathrm{G}$, et al. Blocking angiotensin II Type 1 receptor triggers apoptotic cell death in human pancreatic cancer cells. Pancreas. 2010;39(5):581-94. Epub 2010/02/02.

17. Attoub S, Gaben AM, Al-Salam S, et al. Captopril as a potential inhibitor of lung tumor growth and metastasis. Annals of the New York Academy of Sciences. 2008;1138:65-72. Epub 2008/10/08.

18. Willis LM, El-Remessy $A B$, Somanath PR, et al. Angiotensin receptor blockers and angiogenesis: clinical and experimental evidence. Clinical science (London, England : 1979). 2011;120(8):307-19. Epub 2011/04/14.

19. Huang MM, Guo AB, Sun JF, et al. Angiotensin II promotes the progression of human gastric cancer. Mol Med Rep. 2014;9(3):1056-60.

20. Okazaki M, Fushida S, Harada S, et al. The angiotensin II type 1 receptor blocker candesartan suppresses proliferation and fibrosis in gastric cancer. Cancer letters. 2014;355(1):46-53. Epub 2014/09/17.

21. Bregonzio C, Armando I, Ando $\mathrm{H}$, et al. Anti-inflammatory effects of angiotensin II AT1 receptor antagonism prevent stress-induced gastric injury. American journal of physiology Gastrointestinal and liver physiology. 2003;285(2):G414-23. Epub 2003/04/11.

22. Kinoshita J, Fushida S, Harada $\mathrm{S}$, et al. Local angiotensin II-generation in human gastric cancer: Correlation with tumor progression through the activation of ERK1/2, NF-kappa B and survivin. Int J Oncol. 2009;34(6):1573-82.

23. Wang L, Cai SR, Zhang $\mathrm{CH}$, et al. Effects of angiotensin-converting enzyme inhibitors and angiotensin II type 1 receptor blockers on lymphangiogenesis of gastric cancer in a nude mouse model. Chin Med J. 2008;121(21):2167-71.

24. Bangalore S, Kumar S, Kjeldsen SE, et al. Antihypertensive drugs and risk of cancer: network meta-analyses and trial sequential analyses of 324,168 participants from randomised trials. The Lancet Oncology. 2011;12(1):65-82. Epub 2010/12/03.

25. Yoon C, Yang HS, Jeon I, et al. Use of angiotensin-converting-enzyme inhibitors or angiotensin-receptor blockers and cancer risk: a meta-analysis of observational studies. Can Med Assoc J. 2011;183(14):E1073-E84.

26. Mc Menamin UC, Murray L, Cantwell MM, et al. Angiotensin-converting enzyme inhibitors and angiotensin receptor blockers in cancer progression and survival: a systematic review. Cancer Causes Control. 2012;23(2):221-30.

27. Song $\mathrm{T}$, Choi $\mathrm{CH}$, Kim $\mathrm{MK}$, et al. The effect of angiotensin system inhibitors (angiotensinconverting enzyme inhibitors or angiotensin receptor blockers) on cancer recurrence and survival: a meta-analysis. European journal of cancer prevention : the official journal of the European Cancer Prevention Organisation (ECP). 2017;26(1):78-85. Epub 2016/05/10.

28. Marthey L, Cadiot G, Seksik P, et al. Olmesartan-associated enteropathy: results of a national survey. Alimentary pharmacology \& therapeutics. 2014;40(9):1103-9. Epub 2014/09/10.

29. Herrett E, Gallagher AM, Bhaskaran K, et al. Data Resource Profile: Clinical Practice Research Datalink (CPRD). International journal of epidemiology. 2015;44(3):827-36. Epub 2015/06/08.

30. Khan NF, Perera R, Harper S, et al. Adaptation and validation of the Charlson Index for Read/OXMIS coded databases. BMC family practice. 2010;11:1. Epub 2010/01/07. 
31. Joint Formulary Committee. Joint Formulary Committee. British National Formulary. [BNF online]. $2016 \quad$ [cited 2017 13/04/17]; Available from: https://www.medicinescomplete.com/mc/bnflegacy/current/PHP1219-losartan-potassium.htm.

32. Tamim H, Monfared AA, LeLorier J. Application of lag-time into exposure definitions to control for protopathic bias. Pharmacoepidemiology and drug safety. 2007;16(3):250-8. Epub $2007 / 01 / 25$

33. Chubak J, Boudreau DM, Wirtz HS, et al. Threats to validity of nonrandomized studies of postdiagnosis exposures on cancer recurrence and survival. J Natl Cancer Inst. 2013;105(19):145662. Epub 2013/08/14.

34. Nielsen SF, Nordestgaard BG, Bojesen SE. Statin Use and Reduced Cancer-Related Mortality. N Engl J Med. 2012;367(19):1792-802.

35. Elwood PC, Morgan G, Pickering JE, et al. Aspirin in the Treatment of Cancer: Reductions in Metastatic Spread and in Mortality: A Systematic Review and Meta-Analyses of Published Studies. PLoS One. 2016;11(4):25.

36. Simon R, Makuch RW. A non-parametric graphical representation of the relationship between survival and the occurrence of an event: application to responder versus non-responder bias. Statistics in medicine. 1984;3(1):35-44. Epub 1984/01/01.

37. Lévesque LE, Hanley JA, Kezouh A, et al. Problem of immortal time bias in cohort studies: example using statins for preventing progression of diabetes. BMJ (Clinical research ed). 2010;340.

38. Lund JL, Richardson DB, Stürmer T. The active comparator, new user study design in pharmacoepidemiology: historical foundations and contemporary application. Current epidemiology reports. 2015;2(4):221-8.

39. Lipsitch M, Tchetgen ET, Cohen T. Negative Controls: A Tool for Detecting Confounding and Bias in Observational Studies. Epidemiology (Cambridge, Mass). 2010;21(3):383-8.

40. White IR, Royston P, Wood AM. Multiple imputation using chained equations: Issues and guidance for practice. Statistics in medicine. 2011;30(4):377-99. Epub 2011/01/13.

41. Fine JP, Gray RJ. A Proportional Hazards Model for the Subdistribution of a Competing Risk. Journal of the American Statistical Association. 1999;94(446):496-509.

42. Steele RJC, Brewster DH. Should we use total mortality rather than cancer specific mortality to judge cancer screening programmes? No. BMJ (Clinical research ed). 2011;343.

43. Sarfati D, Blakely T, Pearce N. Measuring cancer survival in populations: relative survival vs cancer-specific survival. International journal of epidemiology. 2010;39(2):598-610. Epub 2010/02/10.

44. Shrank WH, Patrick AR, Alan Brookhart M. Healthy User and Related Biases in Observational Studies of Preventive Interventions: A Primer for Physicians. Journal of General Internal Medicine. 2011;26(5):546-50.

45. White IR, Royston P, Wood AM. Multiple imputation using chained equations: Issues and guidance for practice. Statistics in medicine. 2011;30(4):377-99.

46. Chen $\mathrm{YH}$, Huang $\mathrm{CH}$, Lu HI, et al. Prognostic impact of renin-angiotensin system blockade in esophageal squamous cell carcinoma. Journal of the renin-angiotensin-aldosterone system : JRAAS. 2015;16(4):1185-92. Epub 2014/06/26. 
47. Kim ST, Park KH, Oh SC, et al. How Does Inhibition of the Renin-Angiotensin System Affect the Prognosis of Advanced Gastric Cancer Patients Receiving Platinum-Based Chemotherapy? Oncology. 2012;83(6):354-60.

48. Sugimoto $M$, Yamaoka $Y$, Shirai $N$, et al. Role of renin-angiotensin system in gastric oncogenesis. J Gastroenterol Hepatol. 2012;27(3):442-51.

49. Miwa $\mathrm{H}$, Hongo $\mathrm{M}$, Kusano $\mathrm{M}$. Combination of angiotensin II receptor blockers promotes proton pump inhibitor-based healing of reflux esophagitis. Journal of gastroenterology. 2012;47(3):249-55. Epub 2011/11/02.

50. Joint Formulary Committee. Losartan. In: Joint Formulary Committee. British National Formulary.[BNF online]. 2016 [cited 2017 13/04/17]; Available from: https://www.medicinescomplete.com/mc/bnflegacy/current/PHP1219-losartan-potassium.htm.

51. Sipahi I, Debanne SM, Rowland DY, et al. Angiotensin-receptor blockade and risk of cancer: meta-analysis of randomised controlled trials. The Lancet Oncology. 2010;11(7):627-36. Epub 2010/06/15.

52. Corrao G, Zambon A, Parodi A, et al. Discontinuation of and changes in drug therapy for hypertension among newly-treated patients: a population-based study in Italy. Journal of hypertension. 2008;26(4):819-24. Epub 2008/03/11.

53. Hill AB. The Environment and Disease: Association or Causation? Proceedings of the Royal Society of Medicine. 1965;58(5):295-300. 


\section{Tables and Figures}

Table 1: Patient characteristics by ARB use at any time after diagnosis

\begin{tabular}{|c|c|c|}
\hline & Non-user & User \\
\hline Number of Patients & 4,764 & 360 \\
\hline \multicolumn{3}{|l|}{ Year of Diagnosis } \\
\hline $1998-2002$ & $1,163(24.4 \%)$ & $38(10.6 \%)$ \\
\hline $2003-2007$ & $1,651(34.7 \%)$ & $120(33.3 \%)$ \\
\hline $2008-2012$ & $1,950(40.9 \%)$ & $202(56.1 \%)$ \\
\hline Age at Diagnosis (SD) & 69.5 (11.9) & $71.9(9.3)$ \\
\hline $0-49$ & $268(5.6 \%)$ & $3(0.8 \%)$ \\
\hline $50-59$ & $684(14.4 \%)$ & $33(9.2 \%)$ \\
\hline $60-69$ & $1,284(27.0 \%)$ & $104(28.9 \%)$ \\
\hline $70-79$ & $1,512(31.7 \%)$ & $149(41.4 \%)$ \\
\hline $80+$ & $1,016(21.3 \%)$ & 71 (19.7\%) \\
\hline \multicolumn{3}{|l|}{ Gender } \\
\hline Male & $3,243(68.1 \%)$ & $216(60.0 \%)$ \\
\hline Female & $1,521(31.9 \%)$ & $144(40.0 \%)$ \\
\hline \multicolumn{3}{|l|}{ Deprivation Quintile } \\
\hline 1 (Least Deprived) & 949 (19.9\%) & $74(20.6 \%)$ \\
\hline 2 & $1,182(24.8 \%)$ & $90(25.0 \%)$ \\
\hline 3 & $951(20.0 \%)$ & 71 (19.7\%) \\
\hline 4 & $955(20.1 \%)$ & $78(21.7 \%)$ \\
\hline 5 (Most Deprived) & 723 (15.2\%) & 47 (13.1\%) \\
\hline Missing & 4 & 0 \\
\hline \multicolumn{3}{|l|}{ Comorbidities } \\
\hline Diabetes & 535 (11.2\%) & $101(28.1 \%)$ \\
\hline Chronic pulmonary disease & $572(12.0 \%)$ & $57(15.8 \%)$ \\
\hline Renal disease & $347(7.3 \%)$ & 65 (18.1\%) \\
\hline Cerebrovascular disease & $242(5.1 \%)$ & $18(5.0 \%)$ \\
\hline Peptic ulcer disease & $231(4.8 \%)$ & $18(5.0 \%)$ \\
\hline Peripheral vascular disease & $218(4.6 \%)$ & $25(6.9 \%)$ \\
\hline Myocardial infarction & $172(3.6 \%)$ & $26(7.2 \%)$ \\
\hline Congestive heart disease & $168(3.5 \%)$ & $30(8.3 \%)$ \\
\hline Liver Disease & $22(0.5 \%)$ & $4(1.1 \%)$ \\
\hline \multicolumn{3}{|l|}{ Confounder Medications } \\
\hline Statin & $1,149(24.1 \%)$ & $198(55.0 \%)$ \\
\hline Aspirin & 931 (19.5\%) & $154(42.8 \%)$ \\
\hline \multicolumn{3}{|l|}{ Treatment } \\
\hline Surgery & $2,128(44.7 \%)$ & $186(51.7 \%)$ \\
\hline Chemotherapy & $2,028(42.6 \%)$ & $160(44.4 \%)$ \\
\hline Radiotherapy & 805 (16.9\%) & $64(17.8 \%)$ \\
\hline \multicolumn{3}{|l|}{ Grade } \\
\hline 1 & $183(5.2 \%)$ & 15 (5.9\%) \\
\hline 2 & $1,398(39.8 \%)$ & $109(42.7 \%)$ \\
\hline 3 & $1,900(54.0 \%)$ & $129(50.6 \%)$ \\
\hline 4 & $35(1.0 \%)$ & $2(0.8 \%)$ \\
\hline Missing & 1,248 & 105 \\
\hline \multicolumn{3}{|l|}{ Tumour type } \\
\hline Adenocarcinoma & $3,275(68.7 \%)$ & $265(73.8 \%)$ \\
\hline Squamous & 804 (16.9\%) & 45 (12.5\%) \\
\hline Other & $685(14.4 \%)$ & 49 (13.6\%) \\
\hline \multicolumn{3}{|l|}{ Stage } \\
\hline 1 & $73(9.4 \%)$ & $11(16.9 \%)$ \\
\hline 2 & 147 (19.0\%) & $13(20.0 \%)$ \\
\hline
\end{tabular}




\begin{tabular}{lcc}
3 & $280(36.2 \%)$ & $25(38.5 \%)$ \\
4 & $274(35.4 \%)$ & $16(24.6 \%)$ \\
Missing & 3,990 & 295 \\
Smoking & & \\
Never & $1,707(39.4 \%)$ & $156(45.0 \%)$ \\
Former & $1,557(35.9 \%)$ & $160(46.1 \%)$ \\
Current & $1,069(24.7 \%)$ & $31(8.9 \%)$ \\
Missing & 431 & 13 \\
Alcohol (units per week) & & \\
None & $821(30.1 \%)$ & $71(30.5 \%)$ \\
$1-14$ & $1,366(50.0 \%)$ & $117(50.2 \%)$ \\
$15-28$ & $336(12.3 \%)$ & $31(13.3 \%)$ \\
$29-42$ & $115(4.2 \%)$ & $10(4.3 \%)$ \\
$43+$ & $94(3.4 \%)$ & $4(1.7 \%)$ \\
Missing & 2,032 & 127 \\
BMI (kg/m $\left.{ }^{2}\right)$ & & \\
Underweight $(<18.5)$ & $152(4.4 \%)$ & $2(0.6 \%)$ \\
Normal $(18.5-24.9)$ & $1,239(36.0 \%)$ & $80(25.4 \%)$ \\
Overweight $(25-29.9)$ & $1,352(39.3 \%)$ & $132(41.9 \%)$ \\
Obese $(\geq 30)$ & $698(20.3 \%)$ & $101(32.1 \%)$ \\
Missing & 1,323 & 45 \\
\hline
\end{tabular}


Table 2: Association between ARB use and gastro-oesophageal cancer mortality

\begin{tabular}{|c|c|c|c|c|c|c|}
\hline & $\mathbf{N}$ & Person-Years & Deaths & Unadjusted HR & Adjusted HR ${ }^{\mathrm{a}}$ & Trend p-value \\
\hline \multicolumn{7}{|c|}{ Gastro-oesophageal } \\
\hline \multicolumn{7}{|c|}{ All ARBs Combined } \\
\hline Never & 4,764 & 9,241 & 3,175 & Ref & Ref & 0.003 \\
\hline Ever & 360 & 786 & 170 & $0.80(0.69,0.94)$ & $0.83(0.71,0.98)$ & \\
\hline 1-182 DDDs & 146 & 263 & 92 & $0.87(0.71,1.07)$ & $0.91(0.74,1.13)$ & \\
\hline 183-364 DDDs & 67 & 130 & 40 & $0.92(0.67,1.25)$ & $0.97(0.71,1.33)$ & \\
\hline 365-729 DDDs & 57 & 109 & 24 & $0.82(0.55,1.23)$ & $0.83(0.55,1.25)$ & \\
\hline 730+ DDDs & 90 & 284 & 14 & $0.42(0.25,0.71)$ & $0.42(0.25,0.72)$ & \\
\hline \multicolumn{7}{|l|}{ Losartan } \\
\hline Never & 4,973 & 9,698 & 3,278 & Ref & Ref & \\
\hline Ever & 151 & 330 & 67 & $0.82(0.64,1.04)$ & $0.82(0.64,1.06)$ & \\
\hline \multicolumn{7}{|l|}{ Candesartan } \\
\hline Never & 5,013 & 9,772 & 3,300 & Ref & Ref & \\
\hline Ever & 111 & 255 & 45 & $0.72(0.54,0.97)$ & $0.71(0.52,0.95)$ & \\
\hline \multicolumn{7}{|c|}{ Oesophageal } \\
\hline \multicolumn{7}{|c|}{ All ARBs Combined } \\
\hline Never & 2,565 & 4,499 & 1,776 & Ref & Ref & 0.135 \\
\hline Ever & 168 & 338 & 91 & $0.89(0.72,1.09)$ & $0.89(0.71,1.10)$ & \\
\hline 1-182 DDDs & 68 & 100 & 47 & $0.87(0.65,1.16)$ & $0.89(0.66,1.19)$ & \\
\hline 183-364 DDDs & 31 & 53 & 24 & $1.21(0.80,1.81)$ & $1.21(0.80,1.81)$ & \\
\hline 365-729 DDDs & 30 & 53 & 13 & $0.95(0.55,1.65)$ & $0.92(0.53,1.59)$ & \\
\hline $730+$ DDDs & 39 & 133 & 7 & $0.46(0.22,0.97)$ & $0.44(0.21,0.94)$ & \\
\hline \multicolumn{7}{|l|}{ Losartan } \\
\hline Never & 2,667 & 4,698 & 1,836 & Ref & Ref & \\
\hline Ever & 66 & 139 & 31 & $0.82(0.58,1.17)$ & $0.84(0.59,1.21)$ & \\
\hline \multicolumn{7}{|l|}{ Candesartan } \\
\hline Never & 2,681 & 4,732 & 1,842 & Ref & Ref & \\
\hline Ever & 52 & 105 & 25 & $0.76(0.52,1.14)$ & $0.73(0.49,1.09)$ & \\
\hline \multicolumn{7}{|c|}{ Gastric } \\
\hline \multicolumn{7}{|c|}{ All ARBs Combined } \\
\hline Never & 2,199 & 4,743 & 1,399 & Ref & Ref & 0.009 \\
\hline Ever & 192 & 448 & 79 & $0.75(0.60,0.94)$ & $0.79(0.62,1.00)$ & \\
\hline 1-182 DDDs & 78 & 163 & 45 & $0.89(0.66,1.21)$ & $0.95(0.70,1.28)$ & \\
\hline 183-364 DDDs & 36 & 78 & 16 & $0.70(0.43,1.15)$ & $0.75(0.46,1.24)$ & \\
\hline 365-729 DDDs & 27 & 56 & 11 & $0.74(0.41,1.34)$ & $0.76(0.42,1.39)$ & \\
\hline 730+ DDDs & 51 & 151 & 7 & $0.40(0.19,0.85)$ & $0.40(0.19,0.84)$ & \\
\hline \multicolumn{7}{|l|}{ Losartan } \\
\hline Never & 2,306 & 5,000 & 1,442 & Ref & Ref & \\
\hline Ever & 85 & 191 & 36 & $0.85(0.61,1.18)$ & $0.81(0.57,1.14)$ & \\
\hline \multicolumn{7}{|l|}{ Candesartan } \\
\hline Never & 2,332 & 5,041 & 1,458 & Ref & Ref & \\
\hline Ever & 59 & 150 & 20 & $0.68(0.44,1.06)$ & $0.67(0.43,1.05)$ & \\
\hline
\end{tabular}

${ }^{a}$ Adjusted for age, deprivation, year of diagnosis, cancer site, cancer treatment within 6 months (radiotherapy, chemotherapy, surgery), comorbidities (cerebrovascular disease, chronic pulmonary disease, congestive heart disease, diabetes, liver disease, myocardial infarction, peptic ulcer disease, peripheral vascular disease, renal disease) and other medication use (statins and aspirin, time-varying after diagnosis) 
Figure 1: Association between ARB use and gastro-oesophageal cancer mortality

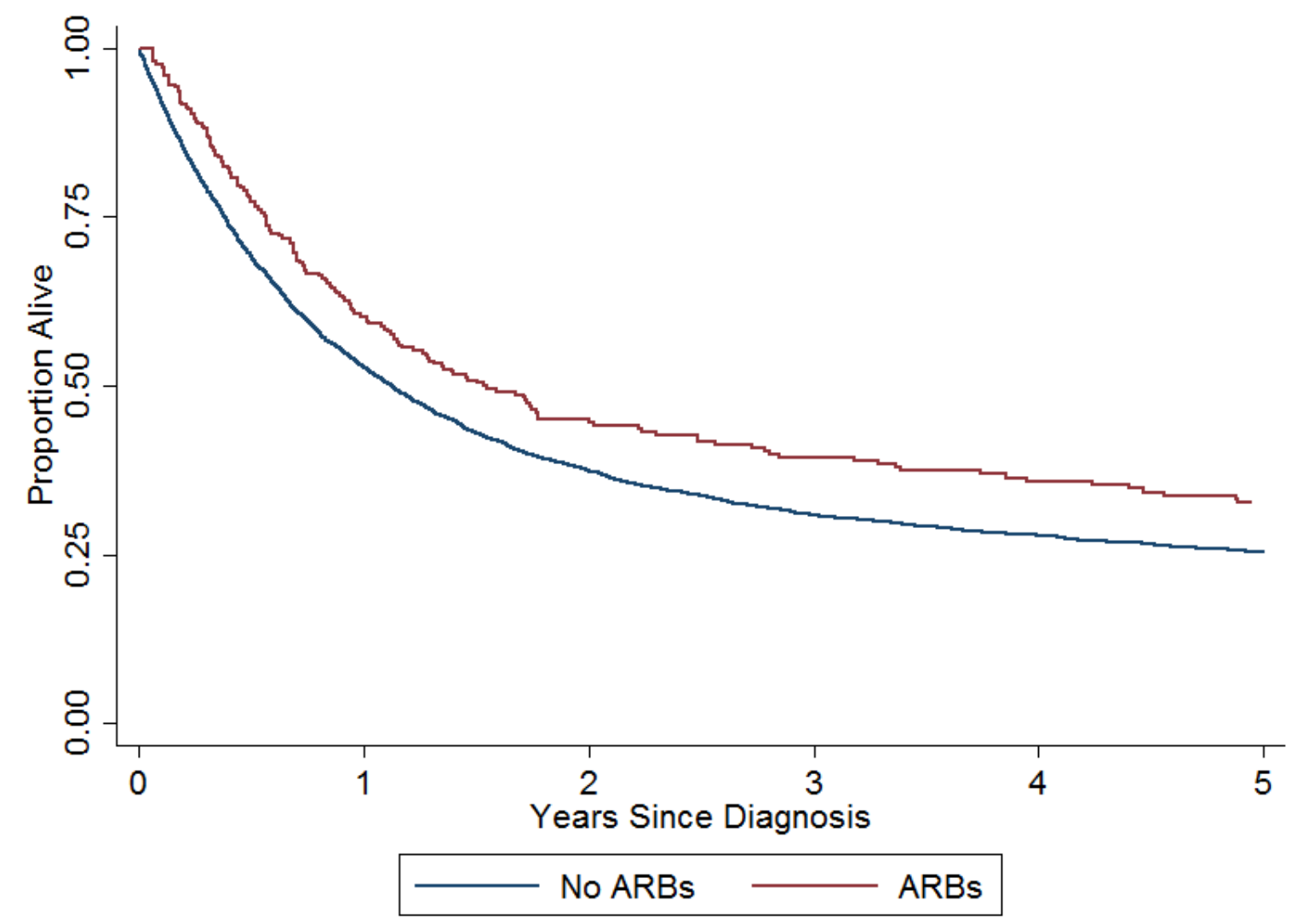


Table 3: Sensitivity and subgroup analysis for ARB use and gastro-oesophageal cancer mortality

\begin{tabular}{|c|c|c|c|c|c|c|c|c|}
\hline & \multicolumn{3}{|c|}{ Non-Users $^{\mathrm{a}}$} & \multicolumn{3}{|c|}{ Users } & \multirow{2}{*}{ Unadjusted HR } & \multirow{2}{*}{ Adjusted HR } \\
\hline & $\mathbf{N}$ & Person-Years & Deaths $^{\mathrm{b}}$ & $\mathbf{N}$ & Person-Years & Deaths & & \\
\hline Main analysis & 4,764 & 9,241 & 3,175 & 360 & 786 & 170 & $0.80(0.69,0.94)$ & $0.83(0.71,0.98)$ \\
\hline \multicolumn{9}{|l|}{ Death definition } \\
\hline All-cause & 4,764 & 9,241 & 3,605 & 360 & 786 & 214 & $0.87(0.76,1.00)$ & $0.87(0.75,1.00)$ \\
\hline Primary or secondary GO cause & 4,764 & 9,241 & 3,350 & 360 & 786 & 189 & $0.84(0.73,0.98)$ & $0.86(0.74,1.00)$ \\
\hline \multicolumn{9}{|l|}{ Exposure definition } \\
\hline Year before diagnosis & 4,757 & 11,733 & 3,130 & 367 & 821 & 215 & $0.91(0.79,1.04)$ & $0.91(0.79,1.05)$ \\
\hline Six months after diagnosis & 4,804 & 9,447 & 3,167 & 320 & 581 & 178 & $0.82(0.71,0.96)$ & $0.85(0.73,1.00)$ \\
\hline \multicolumn{9}{|l|}{ Exposure lag } \\
\hline None & 4,731 & 11,579 & 3,149 & 393 & 976 & 196 & $0.82(0.71,0.95)$ & $0.85(0.73,0.99)$ \\
\hline 12 months & 4,870 & 7,314 & 1,722 & 254 & 629 & 94 & $0.81(0.66,1.00)$ & $0.82(0.66,1.02)$ \\
\hline \multicolumn{9}{|l|}{ Tumour type $^{d}$} \\
\hline Adenocarcinoma & 3,275 & 6,486 & 2,196 & 265 & 596 & 125 & $0.79(0.66,0.94)$ & $0.78(0.65,0.94)$ \\
\hline Squamous cell carcinoma & 804 & 1,305 & 552 & 45 & 78 & 28 & $0.98(0.67,1.43)$ & $0.95(0.63,1.43)$ \\
\hline Surgical treatment & 2,128 & 5,817 & 1,233 & 186 & 527 & 71 & $0.83(0.66,1.06)$ & $0.81(0.63,1.05)$ \\
\hline \multicolumn{9}{|l|}{ Hypertension diagnosis / treatment } \\
\hline Pre-diagnosis hypertension diagnosis ${ }^{\mathrm{e}}$ & 731 & 1,168 & 499 & 116 & 214 & 61 & $0.85(0.65,1.12)$ & $0.83(0.62,1.09)$ \\
\hline Pre-diagnosis antihypertensive medication users ${ }^{f}$ & 2,324 & 3,888 & 1,565 & 335 & 724 & 161 & $0.74(0.63,0.87)$ & $0.80(0.67,0.94)$ \\
\hline ARB vs. other antihypertensive medication ${ }^{\mathrm{g}}$ & 4,764 & 9,241 & 3,175 & 360 & 786 & 170 & $0.80(0.69,0.94)$ & $0.83(0.71,0.98)$ \\
\hline \multicolumn{9}{|l|}{ Multiple Imputation } \\
\hline Lifestyle factors $^{\mathrm{h}}$ & 4,764 & 9,241 & 3,175 & 360 & 786 & 170 & $0.80(0.69,0.94)$ & $0.85(0.73,1.00)$ \\
\hline Tumour prognostic factors ${ }^{i}$ & 4,764 & 9,241 & 3,175 & 360 & 786 & 170 & $0.80(0.69,0.94)$ & $0.87(0.73,1.04)$ \\
\hline Lifesyle \& tumour prognostic factors ${ }^{j}$ & 4,764 & 9,241 & 3,175 & 360 & 786 & 170 & $0.80(0.69,0.94)$ & $0.87(0.73,1.03)$ \\
\hline Competing risks regression ${ }^{k}$ & 4,764 & 9,241 & 3,175 & 360 & 786 & 170 & $0.80(0.69,0.93)$ & $0.84(0.72,0.99)$ \\
\hline
\end{tabular}

a Non-users for each sensitivity analysis except for 'ARB vs. other antihypertensive medication' where patients who received a different antihypertensive medication serve as the non-user group

${ }^{\mathrm{b}}$ Deaths with an underlying cause of gastro-oesophageal cancer unless otherwise stated

${ }^{\mathrm{C}}$ Adjusted for age, deprivation, year of diagnosis, cancer site, cancer treatment within 6 months (radiotherapy, chemotherapy, surgery), comorbidities (cerebrovascular disease, chronic

pulmonary disease, congestive heart disease, diabetes, liver disease, myocardial infarction, peptic ulcer disease, peripheral vascular disease, renal disease) and other medication use (statins, aspirin, time-varying after diagnosis)

d P-value for interaction: 0.518

e Restricted to patients with a hypertension diagnosis (Read code categories $\mathrm{G} 20$ and 662 ) in the year prior to diagnosis 
${ }^{f}$ Restricted to patients with a prescription of any antihypertensive medication (diuretics, vasodilator antihypertensive drugs, centrally acting antihypertensive drugs, alpha-adrenoceptor blocking drugs, beta-blockers, ACEIs, ARBs, renin inhibitors, and calcium channel blockers) in the year prior to cancer diagnosis

$\mathrm{g}$ Using other antihypertensive medications as an active comparator

${ }^{\mathrm{h}}$ Additionally adjusted for smoking, BMI and alcohol consumption

'Additionally adjusted for stage and grade

jAdditional adjusted for smoking, BMI, alcohol consumption, stage and grade

k Using the Fine and Gray sub distribution hazard model with non-gastro-oesophageal cancer death as competing risk 


\section{Appendices}

Appendix 1: List of generic and proprietary drug names used to identify ARBs

\begin{tabular}{ll}
\hline Substance & Medication \\
\hline Azilsartan Medoxomil & Azilsartan, Edarbi (Takeda, High Wycombe, UK) \\
Candesartan Cilexetil & Candesartan, Amias (Takeda, High Wycombe, UK) \\
Eprosartan & Eprosartan, Teveten (Abbott Healthcare, Maidenhead, UK) \\
Irbesartan & Irbesartan, Aprovel (Bristol-Myers Squibb, New York, US \& Sanofi-Aventis, \\
& Gentilly, France), Coaprovel (Bristol-Myers Squibb, New York, US \& Sanofi- \\
& Aventis, Gentilly, France) \\
Losartan Potassium & Losartan, Cozaar (MSD, Hertfordshire, UK) \\
Olmesartan & Olmesartan, Olmetec (Daiichi Sankyo, Tokyo, Japan), Sevikar (Daiichi \\
Medoxomil & Sankyo, Tokyo, Japan) \\
Telmisartan & Telmisartan, Micardis (Boehringer Ingelheim, Rhein, Germany) \\
Valsartan & Valsartan, Diovan (Novartis, Basal, Switzerland), Entresto (Novartis, Basal, \\
& Switzerland) \\
\hline
\end{tabular}


Appendix 2: Illustration of study design for selected analyses ${ }^{a}$

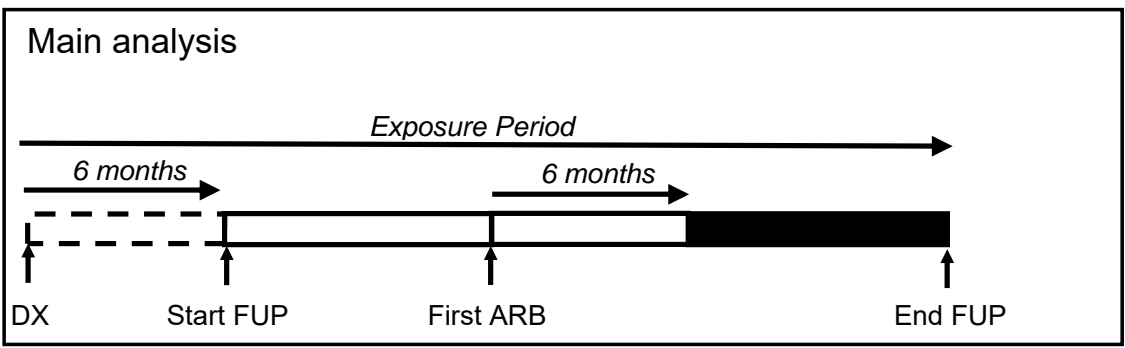

Sensitivity analysis: No exposure lag.

Exposure Period

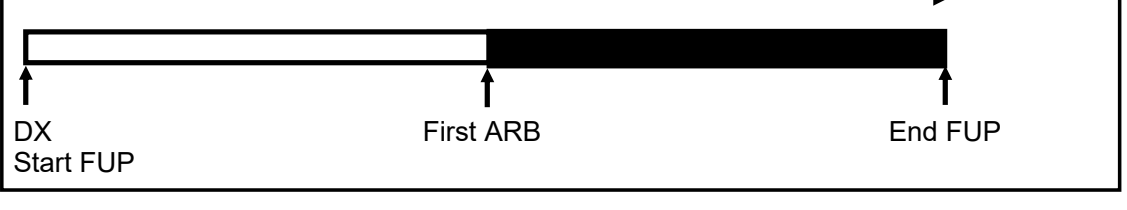

Sensitivity analysis: 12 month exposure lag.

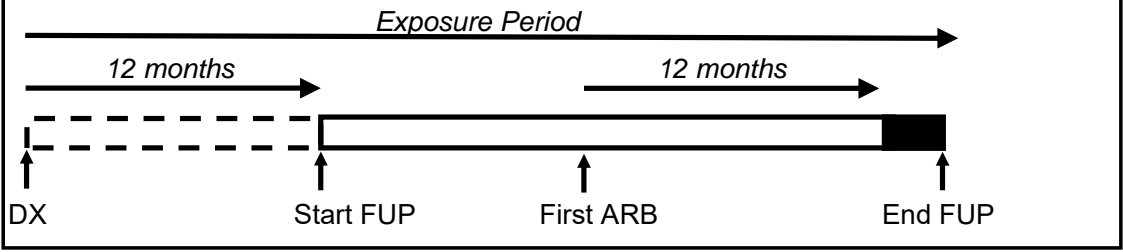

Sensitivity analysis: Year before diagnosis
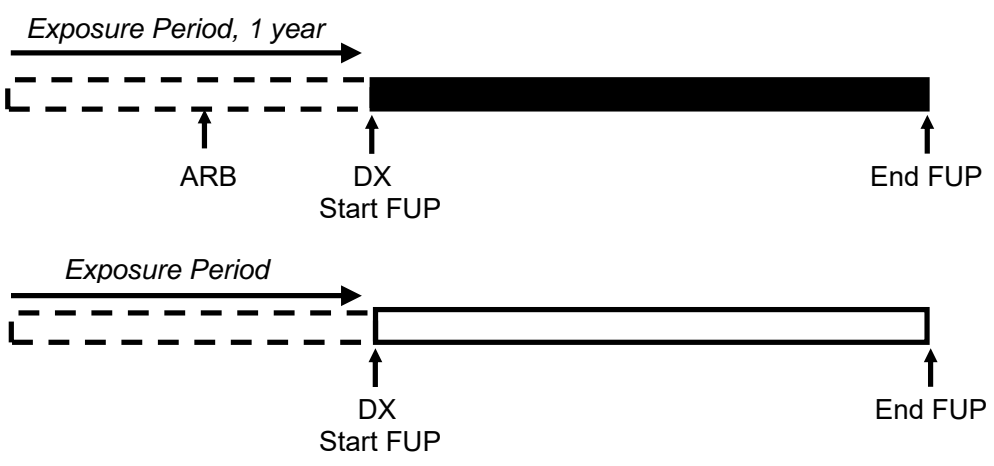

Sensitivity analysis: 6 months after diagnosis

$\underset{\mathrm{DX}}{\stackrel{\text { Exposure Period, } 6 \text { months }}{\mathrm{L}}}$

$\begin{array}{lll}\text { DX } & \text { ARB } & \text { Start FUP }\end{array}$

Exposure Period

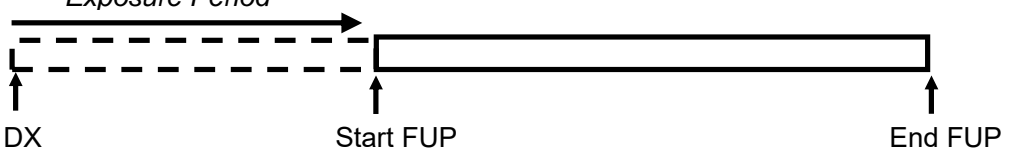

Legend: $\mathbf{L}-\mathbf{I}$ Before FUP $\square$ ARB User $\square$ ARB Non-user

a FUP: follow-up period; DX: breast cancer diagnosis 
Appendix 3: Complete model results for fully-adjust gastro-oesophageal analysis

\begin{tabular}{|c|c|}
\hline Covariate & HR $(95 \% \mathrm{Cl})$ \\
\hline \multicolumn{2}{|l|}{ ARB use } \\
\hline Never & Ref \\
\hline Ever & $0.83(0.71,0.98)$ \\
\hline Age at Diagnosis & $1.02(1.01,1.02)$ \\
\hline \multicolumn{2}{|l|}{ Deprivation Quintile } \\
\hline 1 (Least Deprived) & Ref \\
\hline 2 & $1.07(0.96,1.18)$ \\
\hline 3 & $1.02(0.92,1.14)$ \\
\hline 4 & $1.06(0.95,1.18)$ \\
\hline 5 (Most Deprived) & $1.11(0.99,1.25)$ \\
\hline \multicolumn{2}{|l|}{ Year of Diagnosis } \\
\hline 1998 & Ref \\
\hline 1999 & $0.85(0.66,1.11)$ \\
\hline 2000 & $0.96(0.76,1.22)$ \\
\hline 2001 & $0.77(0.62,0.97)$ \\
\hline 2002 & $0.81(0.65,1.01)$ \\
\hline 2003 & $0.78(0.62,0.97)$ \\
\hline 2004 & $0.69(0.55,0.86)$ \\
\hline 2005 & $0.78(0.62,0.97)$ \\
\hline 2006 & $0.72(0.58,0.90)$ \\
\hline 2007 & $0.67(0.54,0.84)$ \\
\hline 2008 & $0.72(0.58,0.90)$ \\
\hline 2009 & $0.55(0.43,0.69)$ \\
\hline 2010 & $0.54(0.43,0.68)$ \\
\hline 2011 & $0.59(0.46,0.74)$ \\
\hline 2012 & $0.64(0.51,0.81)$ \\
\hline 2013 & $0.55(0.42,0.71)$ \\
\hline \multicolumn{2}{|l|}{ Treatment } \\
\hline Surgery & $0.54(0.50,0.58)$ \\
\hline Chemotherapy & $1.36(1.26,1.47)$ \\
\hline Radiotherapy & $1.10(1.00,1.21)$ \\
\hline \multicolumn{2}{|l|}{ Gender } \\
\hline Male & Ref \\
\hline Female & $0.91(0.85,0.98)$ \\
\hline \multicolumn{2}{|l|}{ Site } \\
\hline Oesophageal & Ref \\
\hline Gastric & $0.89(0.83,0.96)$ \\
\hline \multicolumn{2}{|l|}{ Comorbidities } \\
\hline Cerebrovascular disease & $0.99(0.83,1.19)$ \\
\hline Chronic pulmonary disease & $0.91(0.81,1.03)$ \\
\hline Congestive heart disease & $1.14(0.94,1.40)$ \\
\hline Diabetes & $1.08(0.97,1.22)$ \\
\hline Myocardial infarction & $1.05(0.85,1.28)$ \\
\hline Peptic ulcer disease & $0.73(0.61,0.89)$ \\
\hline Peripheral vascular disease & $1.06(0.89,1.26)$ \\
\hline Renal disease & $1.15(1.00,1.33)$ \\
\hline Liver Disease & $0.82(0.44,1.53)$ \\
\hline \multicolumn{2}{|l|}{ Other medication use } \\
\hline Statin & Not shown ${ }^{a}$ \\
\hline Aspirin & Not shown \\
\hline
\end{tabular}

${ }^{a}$ Hazard ratios for statin and aspirin use are not shown as our protocol states these are to be published separately. 
Appendix 4: Negative control analysis for ACE inhibitor use and gastro-oesophageal cancer mortality

\begin{tabular}{lcccccc}
\hline & N & Person-Years & Deaths & Unadjusted HR & Adjusted HR & Trend P-value $^{\mathbf{a}}$ \\
\hline Gastro-oesophageal & & & & & & \\
$\quad$ Never & 4,061 & 7,916 & 2,746 & Ref & Ref & 0.343 \\
Ever & 1,063 & 2,111 & 599 & $0.96(0.88,1.05)$ & $0.98(0.89,1.08)$ & \\
1-182 DDDs & 384 & 668 & 234 & $0.86(0.75,0.98)$ & $0.86(0.75,0.99)$ & \\
183-364 DDDs & 189 & 321 & 131 & $1.03(0.86,1.23)$ & $1.05(0.87,1.25)$ & \\
365-729 DDDs & 188 & 330 & 119 & $1.15(0.96,1.39)$ & $1.20(0.99,1.45)$ & \\
730+ DDDs & 302 & 792 & 115 & $0.97(0.80,1.17)$ & $1.04(0.85,1.27)$ & \\
Oesophageal & & & & & & \\
Never & 2,182 & 3,914 & 1,526 & Ref & Ref & 0.054 \\
Ever & 551 & 923 & 341 & $1.03(0.92,1.16)$ & $1.04(0.91,1.18)$ & \\
1-182 DDDs & 203 & 322 & 132 & $0.88(0.73,1.05)$ & $0.86(0.71,1.03)$ & \\
183-364 DDDs & 114 & 156 & 80 & $1.12(0.89,1.40)$ & $1.14(0.91,1.45)$ & \\
365-729 DDDs & 103 & 141 & 68 & $1.32(1.03,1.68)$ & $1.35(1.05,1.73)$ & \\
730+ DDDs & 131 & 304 & 61 & $1.10(0.84,1.43)$ & $1.16(0.88,1.52)$ & \\
Gastric & & & & & & \\
Never & 1,879 & 4,002 & 1,220 & $R e f$ & $R .679$ \\
Ever & 512 & 1,188 & 258 & $0.89(0.78,1.02)$ & $0.92(0.79,1.07)$ & \\
1-182 DDDs & 181 & 347 & 102 & $0.84(0.69,1.03)$ & $0.85(0.69,1.05)$ & \\
183-364 DDDs & 75 & 165 & 51 & $0.91(0.69,1.21)$ & $0.93(0.70,1.25)$ & \\
365-729 DDDs & 85 & 189 & 51 & $1.01(0.76,1.34)$ & $1.06(0.79,1.41)$ & \\
730+ DDDs & 171 & 487 & 54 & $0.88(0.67,1.16)$ & $0.95(0.71,1.26)$ & \\
\hline
\end{tabular}

a Adjusted for age, deprivation, year of diagnosis, cancer treatment within 6 months (radiotherapy, chemotherapy, surgery), comorbidities (cerebrovascular disease, chronic pulmonary disease, congestive heart disease, diabetes, liver disease, myocardial infarction, peptic ulcer disease, peripheral vascular disease, renal disease) and other medication use (statins, aspirin, time-varying after diagnosis) 